\title{
PROJETO VINCULAR: ATIVIDADES EDUCATIVAS COM GESTANTES NO CONTEXTO DE UMA MATERNIDADE DO INTERIOR PAULISTA
}

\author{
Lina Domenica Mapelli \\ Universidade Federal de São Carlos \\ mapellilina@gmail.com
}

Giovanna Brunna da Silva Justino Universidade Federal de São Carlos giovanabrunna@gmail.com

Mariana De Gea Gervasio

Universidade de São Paulo marianaggervasio@gmail.com
Jamile de Claro Castro Bussadori

Universidade Federal de São Carlos jamile@ufscar.br

Eliane de Lima Leite Sansão Maternidade Santa Casa de Misericórdia de São Carlos eliane.sansao@santacasasaocarlos.com.br

Natália Rejane Salim Universidade Federal de São Carlos nat.salim@ufscar.br

\section{Resumo}

Trata-se de um relato de experiência de uma atividade de extensão desenvolvida em uma cidade do interior de São Paulo, que teve como objetivo central a aproximação de gestantes à maternidade de referência por meio de estratégias de educação em saúde. A atividade foi realizada através de parceria entre o curso de enfermagem de uma universidade federal e a maternidade. As ações foram fundamentadas na garantia do acesso e do direito a informações de qualidade durante a gestação. No ano de 2019 foram realizados 40 encontros. As estratégias de educação em saúde durante a gestação possibilitaram a aproximação com a maternidade de referência e o contato com temáticas para a preparação para o parto. Projetos como esse são capazes de fortalecer a autonomia das mulheres e possibilitam o compartilhamento de conhecimento contribuindo para o fortalecimento de uma atenção obstétrica digna e respeitosa.

Palavras-chave: Educação em Saúde. Relações Comunidade-instituição. Saúde da Mulher. Serviços de Saúde Materna.

\section{"VINCULAR" PROJECT: GROUP OF PREGNANT WOMEN IN THE CONTEXT OF A MATERNITY IN THE COUNTRYSIDE OF THE STATE OF SÃO PAULO}

\begin{abstract}
This is an experience report of an extension activity developed in a city in the countryside of the state of São Paulo where the main goal was to bring pregnant women closer to the reference maternity through health education strategies. The activity was carried out through a partnership between the nursing course at a federal university and the local maternity hospital. The actions were based on ensuring access and the right to quality information during pregnancy. In the year 2019, 40 meetings were held. Health education strategies during pregnancy made it possible to get closer to the reference maternity and to address topics related to the preparation for childbirth. Projects like this are able to strengthen the autonomy of women and make it possible to share knowledge in order to contribute to the strengthening of a dignified and respectful obstetric care.
\end{abstract}

Keywords: Health Education. Community-institution Relations. Women's Health. Maternal Health Services.

\section{PROYECTO "VINCULAR": GRUPO DE MUJERES EMBARAZADAS EN EL CONTEXTO DE UNA MATERNIDAD EN EL INTERIOR DE PAULISTA}

\section{Resumen}

Es un relato de experiencia de una actividad de extensión desarrollada en una ciudad del interior de São Paulo cuyo principal objetivo fue acercar a la gestante a la maternidad de referencia a través de estrategias de educación en salud. La actividad se realizó mediante una alianza entre el curso de enfermería de una universidad federal y la maternidad. Las acciones se basaron en garantizar el acceso y el derecho a información de calidad durante el embarazo. En el año 2019 se realizaron 40 reuniones. Las estrategias de educación en salud durante el embarazo permitieron acercarse a la maternidad de referencia y contactar temas para la preparación para el parto. Proyectos como este logran fortalecer la autonomía de las mujeres y posibilitar el intercambio de conocimientos para contribuir al fortalecimiento de una atención obstétrica digna, respetuosa y empoderadora.

Palabras clave: Educación para la Salud. Relaciones Comunidad-institución. La Salud de la Mujer. Servicios de Salud Materna. 
Projeto Vincular: atividades educativas com gestantes no contexto de uma maternidade do interior paulista

\section{INTRODUÇÃO}

O movimento organizado de mulheres tem atuado no campo da saúde pública desde a década de 1980, para a inclusão de uma perspectiva da saúde integral da mulher - e a mudança de modelo de atenção obstétrica tem sido umas de suas pautas (PICHETH; CRUBELLATE; VERDU, 2018; BRASIL, 2002). Apenas no início dos anos 2000 surgiu o Programa Nacional de Humanização do Pré-Natal e Nascimento (PHPN) voltado efetivamente para qualidade da assistência ao pré-natal, parto e nascimento, em que se evidenciou a importância da redução da taxa de cesarianas no Brasil, a diminuição de intervenções desnecessárias no parto e a qualificação na cobertura da atenção ao pré-natal, parto e puerpério (PICHETH; CRUBELLATE; VERDU, 2018; BRASIL, 2002).

Em continuidade, com o objetivo de formar uma rede universal, integral e equitativa ao ciclo gravídico-puerperal, estruturando a assistência em saúde do planejamento reprodutivo, prénatal, nascimento e cuidados neonatais, em 2011 o Ministério da Saúde, através da Portaria no 1.459, instituiu a Rede Cegonha (RC) (SAMPAIO et al., 2018; BRASIL, 2011).

Todos esses esforços evidenciaram a luta em prol da garantia dos direitos das mulheres, à atenção respeitosa no decorrer de todo o ciclo gravídico-puerperal; transformando-se em um ponto central a transformação da assistência obstétrica brasileira (PICHETH; CRUBELLATE; VERDU, 2018; BRASIL, 2002).

Neste contexto, as ações das equipes de saúde devem amparar tanto os aspectos clínicos e técnicos do pré-natal, parto e nascimento, quanto os de educação em saúde como potenciais fortalecedores dos direitos das mulheres, e promotores de experiências positivas. Assim, desde o pré-natal deve-se proporcionar espaços de acolhimento e trocas de conhecimentos, aprendizados e preparação para o parto, nascimento e cuidados com recém-nascido (HOLANDA et al., 2018; THEOPHILO; RATTNER; PEREIRA, 2018).

As mulheres devem ter autonomia para escolherem o local do parto e deve haver espaço que possibilite o diálogo diretamente com a equipe sobre procedimentos e condutas ao longo do ciclo gravídico-puerperal (BRASIL, 2002). No entanto, inseguranças se mostram presentes em diversos aspectos, principalmente durante a gestação, com os quais as mulheres e famílias, muitas vezes, não têm a possibilidade de conhecer previamente o local em que acontecerá o nascimento ou com a temática de preparação para o parto (HOLANDA et al., 2018). Desta forma, a educação em saúde pode atuar como estratégia de empoderamento e sensibilização das equipes para melhorias na qualidade da atenção obstétrica (HOLANDA et al., 2018). 
Projeto Vincular: atividades educativas com gestantes no contexto de uma maternidade do interior paulista

As atividades de educação em saúde são fundamentais para o desenvolvimento da corresponsabilização e trocas de conhecimentos de forma horizontalizada, em especial no ciclo gravídico-puerperal, onde as mulheres ocupam o centro do processo educativo (PAIVA; CAETANO, 2020). E a atuação de enfermeiros/as e obstetrizes são essenciais para a garantia desses direitos durante a gestação, bem como nos processos de educação em saúde, com o intuito de possibilitar o compartilhar acerca de dúvidas sobre o desenvolvimento da gestação, a preparação para o parto e nascimento e a prevenção de violência obstétrica (SAMPAIO et al., 2018; BRASIL, 2011).

A rede deve operar como um interlaço efetivo entre atenção primária à saúde e a atenção hospitalar, empregando estratégias que proporcionam o acesso e o vínculo, de forma a fortalecer atuações em conjunto e, com isso, possibilitar a redução e o combate das diferentes formas de desigualdades (PAIVA; CAETANO, 2020; THEOPHILO; RATTNER; PEREIRA. 2018).

Diante do exposto, esse relato é resultado de um projeto de extensão, o Projeto Vincular, desenvolvido por meio de parceria entre o curso de enfermagem da Universidade Federal de São Carlos e a maternidade do município. O objetivo central é compartilhar a experiência da realização desse projeto no ano de 2019 e suas potencialidades para o fortalecimento da rede de cuidado e apoio à gestante, ampliando a atenção à saúde no ciclo gravídico-puerperal.

\section{MATERIAIS E MÉTODOS}

A Maternidade Dona Francisca Cintra Silva, da Irmandade Santa Casa de Misericórdia de São Carlos, São Paulo, é originária da década de 50, conta com 56 leitos, 34 do Sistema Único de Saúde - SUS, 3 particulares e os demais de convênios. Realiza atendimentos de alta complexidade, especializados no ciclo gravídico-puerperal, é única no munícipio e também é referência regional (MATERNIDADE DONA FRANCISCA CINTRA SILVA, 2020).

O Projeto Vincular iniciou-se como extensão universitária em 2017, no núcleo de Saúde da Mulher do Departamento de Enfermagem, Universidade Federal de São Carlos. Seu propósito abrange a construção e solidificação de vínculos entre as gestantes usuárias do SUS, seus acompanhantes/famílias à maternidade, estendendo oportunidades para as usuárias de convênios e particulares também atendidos na instituição. Foram realizadas divulgações nas Unidades de Saúde da Família (USF) e Unidades Básicas de Saúde (UBS) bem como apresentação do projeto ao grupo condutor da rede cegonha do município. Gestantes e acompanhantes de diferentes áreas da cidade participaram do projeto. Os encontros foram oferecidos quinzenalmente e organizados com grupos de cinco a dez participantes, mediante agendamento telefônico ou 
Projeto Vincular: atividades educativas com gestantes no contexto de uma maternidade do interior paulista

pessoalmente. Foi realizado um encontro com cada grupo com apresentação do espaço físico da maternidade, seguido de roda de conversa em sala privativa na própria instituição. O enfoque dos encontros se deu na temática da preparação para o parto, utilizando-se da roda de conversa como principal ferramenta para proporcionar um espaço de troca, fala e escuta. Apesar da atividade ocorrer em um único encontro, foi oferecida a possibilidade de realização de outro agendamento, principalmente para as gestantes que se encontravam no início da gestação. Cada encontro teve a duração média de uma hora e trinta minutos. A atividade foi conduzida por integrantes da equipe do projeto, docentes e discentes em parceria com enfermeiras obstetras e obstetrizes que atuam na instituição.

\section{RESULTADOS E ANÁLISES}

Os encontros foram organizados através de uma apresentação inicial, seguida de uma visita guiada para o reconhecimento do espaço físico da maternidade. Posteriormente, em uma sala adaptada foi desenvolvida a estratégia de rodas de conversa sobre as práticas de saúde com enfoque na preparação para o parto, de forma a garantir um espaço de escuta para as mulheres e seus/suas acompanhantes. Durante os encontros foram utilizados temas disparadores, como, por exemplo, fisiologia e os sinais de trabalho de parto, formas não farmacológicas para o alívio da dor, práticas de cuidado durante o trabalho de parto e o pós-parto imediato. Diante da valorização da participação ativa das/dos participantes em modelo de roda de conversa, temas diferentes também puderam ser discutidos nos encontros conforme a dinâmica de cada grupo, como violência obstétrica, amamentação e depressão pós-parto.

De forma a possibilitar interação e trocas entre as/os participantes foram encorajadas/os a partilharem vivências e tirar as suas dúvidas. Também foram utilizadas estratégias de interações e trabalho corporal como técnicas de relaxamento, dinâmicas sobre o trabalho de parto, formas não farmacológicas de alívio das dores. Nesses momentos as mulheres e acompanhantes foram convidadas/os a experimentar posições, perceber e sentir o próprio corpo. Foram utilizados modelos anatômicos de úteros; bonecos educativos; recursos como aromaterapia (óleos essenciais) e música; difundindo, ainda, possibilidades dos/as participantes experimentarem posições e utilizarem artefatos como bola de ginástica e banqueta de parto.

Foram realizados 40 encontros em 2019, com a participação de 263 pessoas, sendo 137 gestantes e 126 acompanhantes. Das gestantes participantes, 94 realizaram o pré-natal na atenção primária à saúde na rede SUS e 39 estavam associadas a algum tipo de convênio ou atendimento 
Projeto Vincular: atividades educativas com gestantes no contexto de uma maternidade do interior paulista

particular. Duas residiam em municípios vizinhos, uma residia em um distrito rural da região e uma realizava acompanhamentos no Ambulatório de Cuidados Especiais em Gestação (ACEG).

A Rede Cegonha tem em suas diretrizes a vinculação entre atenção primária à saúde e atenção hospitalar, contribuindo para a integralidade do cuidado e ao acesso a informações com uma equânime cobertura do ciclo gravídico-puerperal (PAIVA; CAETANO, 2020; SAMPAIO et al., 2018; BRASIL, 2011). A parceria entre o curso de enfermagem da Universidade Federal de São Carlos e a maternidade possibilitou que gestantes e suas famílias se aproximassem do local de parto preliminarmente, além da oferta de atividades de educação em saúde, que são capazes de corroborar para a efetivação dos direitos das mulheres (PAIVA; CAETANO, 2020; SAMPAIO et al., 2018; BRASIL, 2011).

Assim, a estratégia do diálogo em rodas de conversa e do contato das gestantes à maternidade com possibilidade de aproximação da equipe se mostrou positiva. Essa ação educativa articulada em rede, juntamente com a atenção primária e com ações contínuas, pode ser uma ação potente para o exercício da autonomia e direito das mulheres. Quando se investe na autonomia, no empoderamento e na centralização dos cuidados nas mulheres, olham-se as demandas e necessidades do grupo, rompendo com uma lógica prescritiva (PAIVA; CAETANO, 2020).

Para a efetivação dos direitos de saúde, se faz necessário zelar pela humanização no acolhimento da atenção primária até a atenção hospitalar, com a possibilidade de trocas, garantias de informações sobre todo o ciclo gravídico-puerperal e valorização da mulher (PAIVA; CAETANO, 2020).

As principais dúvidas levantadas durante as rodas de conversa estavam relacionadas aos sinais, fases do trabalho de parto e tipos de parto. Durante os encontros, muitas mulheres e acompanhantes se mostravam surpresos quando foram levantadas temáticas como plano de parto e violência obstétrica, por terem naquele momento o primeiro contato com esses conceitos. Esse espaço possibilitou compartilhar boas práticas na atenção ao parto, baseadas em evidências científicas atuais (BRASIL, 2017).

Inúmeros desafios estão postos para a garantia dos direitos das mulheres (PAIVA; CAETANO, 2020). Pode-se observar a falta de abertura para conversas durante o pré-natal com o enfoque na preparação para o parto e nascimento (DIAS et al., 2019). Questões acerca da cultura da cesariana no país ainda se mostram como uma barreira, constituindo a busca por clarificações a esse respeito (PICHETH; CRUBELLATE; VERDU, 2018; HOLANDA et al., 2018). Algumas participantes e seus acompanhantes partilharam sobre o medo do parto normal e relataram, durante o momento da roda de conversa, experiências negativas prévias, próprias e de 
Projeto Vincular: atividades educativas com gestantes no contexto de uma maternidade do interior paulista

pessoas de suas redes de convívio. Em muitos momentos demostraram ter o interesse restrito ao conhecimento físico do local e as regras da instituição, como: alimentação, horário de visitas, quantidade de materiais de higiene e roupas a serem levadas. Muitas/os consideravam que o projeto se limitava às visitas e se surpreenderam ao serem convidados/as para participar da roda de conversa.

Questões culturais observadas durante os encontros também estiveram relacionadas ao papel dos homens/companheiros no processo de parto e nascimento. Alguns olhares e falas de estranhamento foram manifestados quanto aos assuntos, como a responsabilidade compartilhada com o pai pelos cuidados do recém-nascido. Foi possível identificar que as mulheres ainda ocupam o lugar de únicas ou principais responsáveis pelos cuidados do bebê, os diálogos revelaram posicionamentos enraizados em construções de gênero sobre o papel masculino e feminino, sendo a maternidade muitas vezes ainda vista como natural e inata às mulheres. As diferentes experiências e posicionamentos foram campo para a problematização das questões de gênero e discussão sobre a sobrecarga das mulheres no pós-parto, a importância da rede de apoio e o exercício da paternidade ativa (TEIXEIRA et al., 2015).

Iniciativas como essa permitem uma vinculação à maternidade de forma mais qualificada; para além do conhecimento do espaço físico, regras institucionais, possibilitando novas formas de aproximação e acolhimento. O compartilhar de informações se mostra como uma ação potente para o fortalecimento da autonomia das mulheres (PAIVA; CAETANO, 2020; DIAS et al., 2019; PADILHA et al., 2018). Sabe-se que mulheres que possuem menos possibilidade de se vincularem à maternidade de referência, para conhecerem e compreenderem sobre o seu funcionamento, atravessam maior peregrinação no parto (DIAS et al., 2019).

Desafios foram encontrados no transcorrer desta atividade, estando associados às limitações do ambiente físico e dificuldades para o envolvimento da equipe de saúde da instituição. A falta de espaços para ações como essa dentro do ambiente hospitalar é constantemente colocada como obstáculo para realização de atividades de educação em saúde na atenção secundária. Já a pouca participação da equipe mostrou estar relacionada com as demandas dos plantões e às jornadas de trabalho. No entanto, reconhece-se a disponibilidade da equipe e as adaptações feitas no ambiente físico para o desenvolvimento da atividade. Pode-se afirmar que a parceria entre universidade e maternidade possibilitou mudanças e aproximação com a gestão, o que tornou possível colocar em prática os pressupostos do conceito de ambiência da Política Nacional de Humanização (PNH), formulando e reformulando espaços de encontros dentro das instituições, significando e ressignificando tais espaços para todos/as que fazem parte da rede, de usuárias/famílias e equipes de saúde (PADILHA et al., 2018). 
Projeto Vincular: atividades educativas com gestantes no contexto de uma maternidade do interior paulista

O reconhecimento dessa ação foi percebido no final dos encontros, nas conversas que se estendiam no sentimento de gratidão das/dos participantes e em elogios recebidos através de manifestações, como uma carta de agradecimento entregue na instituição. Durante uma das rodas de conversa, uma das mulheres que havia participado do projeto anteriormente estava no alojamento conjunto com seu bebê e acompanhante. Ela declarou o desejo de participar do encontro e foi convidada a compartilhar a sua experiência de parto e nascimento. Foi um momento muito marcante de interação e de confirmação da importância da iniciativa para as mulheres. A roda de conversa é uma estratégia prevista em políticas de saúde (BRASIL, 2007), entretanto, as dificuldades em reconhecer essa estratégia como uma prática de cuidado refletem na pouca valorização e nos obstáculos em tornar essa iniciativa como uma diretriz institucional.

Torna-se fundamental a reflexão sobre formas diferenciadas de acesso das gestantes aos serviços de saúde; atuando na diminuição do distanciamento e fragmentação do cuidado entre atenção primária à saúde e atenção hospitalar (DIAS et al., 2019; PADILHA et al., 2018). Articulações de diferentes saberes, valores e interesses entre gestores, profissionais de saúde e usuárias/acompanhantes/famílias, beneficiam uma relação mais duradoura e de confiança entre as gestantes e as instituições; além de aperfeiçoar práticas de cuidado fundamentadas na integralidade, atentas às necessidades de saúde desta população e as transformações do modelo de atenção obstétrica (DIAS et al., 2019; PADILHA et al., 2018).

A articulação de diferentes saberes, valores e interesses entre universidade, gestores, profissionais de saúde e usuárias favorece uma relação de confiança, além de sustentar um atendimento integral orientado às necessidades de saúde da população e a transformação de práticas (PADILHA et al., 2018).

\section{CONSIDERAÇÕES FINAIS}

A atenção obstétrica pode ser qualificada através de relações positivas entre a atenção primária à saúde e atenção hospitalar. Ações educativas como essa estão em consonância com as diretrizes da rede cegonha e da garantia dos direitos através do conhecimento do local do parto previamente, a aproximação com a equipe de saúde e a construção de espaços de trocas. Nesse contexto, a universidade deve ter o papel ativo no desenvolvimento de iniciativas e parcerias que a aproximem da comunidade, bem como de toda a rede de atenção à saúde das mulheres.

Uma das limitações dessa atividade foi a realização de encontros pontuais com diferentes grupos de mulheres. Ressalta-se, assim, a importância do desenvolvimento de ações que 
Projeto Vincular: atividades educativas com gestantes no contexto de uma maternidade do interior paulista

possibilitem maior continuidade e vinculação com o mesmo grupo. Entretanto, evidencia-se a importância das ações educativas no período gestacional no contexto hospitalar.

O relato desta experiência propicia o compartilhar de saberes e estratégias de educação em saúde para o cuidado das mulheres, as quais podem ser disseminadas, possibilitando o fortalecimento de uma atenção obstétrica digna, respeitosa e empoderadora. Além disso, demonstra que as articulações e comunicações eficazes entre a atenção primária à saúde e atenção hospitalar resultam em experiências positivas para as mulheres, acompanhantes/famílias e profissionais da saúde do ciclo gravídico-puerperal.

\section{REFERÊNCIAS}

BRASIL. Ministério da Saúde. Portaria no 1.459, de 24 de junho de 2011. Institui no âmbito do Sistema Único de Saúde - SUS - a Rede da Cegonha. Disponível em: https://bvsms.saude.gov.br/bvs/saudelegis/gm/2011/prt1459_24_06_2011.html. Acesso em: 15 jul. 2020.

BRASIL. Ministério da Saúde. Secretaria de Ciência, Tecnologia e Insumos Estratégicos. Departamento de Gestão e Incorporação de Tecnologias em Saúde. Diretrizes nacionais de assistência ao parto normal: versão resumida [recurso eletrônico]. Ministério da Saúde, Secretaria de Ciência, Tecnologia e Insumos Estratégicos, Departamento de Gestão e Incorporação de Tecnologias em Saúde. - Brasília: Ministério da Saúde, 2017. 51p. Disponível em:

http://bvsms.saude.gov.br/bvs/publicacoes/diretrizes_nacionais_assistencia_parto_normal.pdf . Acesso em: 11 set. 2020.

BRASIL. Ministério da Saúde. Secretaria de Gestão Estratégica e Participativa. Departamento de Apoio à Gestão Participativa. Caderno de educação popular e saúde. Ministério da Saúde, Secretaria de Gestão Estratégica e Participativa, Departamento de Apoio à Gestão Participativa. Brasília: Ministério da Saúde, 2007. 160 p. - (Série B. Textos Básicos de Saúde). Disponível: http://bvsms.saude.gov.br/bvs/publicacoes/caderno_educacao_popular_saude_p1.pdf. Acesso em: 11 set. 2020 .

BRASIL. Ministério da Saúde. Secretaria de Políticas de Saúde. Programa de Humanização do Pré-Natal e Nascimento. Brasília (DF): Ministério da Saúde, 2002. 28p. Disponível em: https://bvsms.saude.gov.br/bvs/publicacoes/parto.pdf. Acesso em: 12 jun. 2020.

DIAS, P.F. et al. Formação do conceito ambiência para trabalho de parto e parto normal institucionalizado. Rev. Bras. Enferm., v.72, Suppl.3, p.348-359, 2019. Disponível em: https://www.scielo.br/scielo.php?pid=S0034-71672019000900348\&script=sci_arttext\&tlng=pt. Acesso em: 07 jul. 2020.

HOLANDA, S.M. et al. Influência da participação do companheiro no pré-natal: satisfação de primíparas quanto ao apoio no parto. Texto context-enferm., v.27, n.2, p.01-10, 2018. Disponível em: https://www.scielo.br/scielo.php?script=sci_abstract\&pid=S010407072018000200317\&lng=en\&nrm=iso\&tlng=pt. Acesso em: 12 jun. 2020. 
Projeto Vincular: atividades educativas com gestantes no contexto de uma maternidade do interior paulista

MATERNIDADE DONA FRANCISCA CINTRA SILVA. Santa Casa de Misericórdia de São Carlos, São Paulo, 2020. Disponível em: http://santacasasaocarlos.com.br/page.php?name=maternidade. Acesso em: 07 jul. 2020.

PADILHA, R.Q. et al. Princípios para a gestão da clínica: conectando gestão, atenção à saúde e educação na saúde. Ciênc. saúde coletiva, v.23, n.12, p.4249-4257, 2018. Disponível em: https://www.scielo.br/pdf/csc/v23n12/1413-8123-csc-23-12-4249.pdf. Acesso em: 20 jun. 2020.

PAIVA, C.C.N.; CAETANO, R. Avaliação de implantação das ações de saúde sexual e reprodutiva na Atenção Primária: revisão de escopo. Esc. Anna Nery, v.24, n.1, p.01-11, 2020. Disponível em: http://www.scielo.br/scielo.php?script=sci_arttext\&pid=S141481452020000100703\&lng=en. Acesso em: 07 jul. 2020.

PICHETH, S.F.; CRUBELLATE, J.M.; VERDU, F.C. A transnacionalização do parto normal no Brasil: um estudo das últimas cinco décadas. Hist. cienc. saude-Manguinhos, v.25, n.4, p.10631082, 2018. Disponível em: http://www.scielo.br/scielo.php?script=sci_arttext\&pid=S010459702018000401063\&lng=en.. Acesso em: 12 jun. 2020.

SAMPAIO, L.M. et al. Rede cegonha: acompanhamento pré-natal e vinculação de gestantes à maternidade de referência. Ciência Cuidado e Saúde, v.17, n.1, p.01-07, 2018. Disponível em: http://www.periodicos.uem.br/ojs/index.php/CiencCuidSaude/article/view/38384. Acesso em: 12 jun. 2020.

TEIXEIRA, R.C. et al. Necessidades de saúde de mulheres em pós-parto. Rev. Esc. Anna Nery, v.19, n.4, p.621-628, 2015. Disponível em: https://www.scielo.br/pdf/ean/v19n4/14148145-ean-19-04-0621.pdf . Acesso em: 11 set. 2020.

THEOPHILO, R.L.; RATTNER, D.; PEREIRA, E.L. Vulnerabilidade de mulheres negras na atenção ao pré-natal e ao parto no SUS: análise da pesquisa da Ouvidoria Ativa. Ciênc. saúde coletiva, v.23, n.11, p. 3505-3516, 2018. Disponível em: https://www.scielo.br/scielo.php?script=sci_abstract\&pid=S141381232018001103505\&lng=pt\&nrm=iso . Acesso em: 12 jun. 2020.

Recebido em: 02/10/2020

Aceito em: 14/08/2021 